\title{
Incidence and outcome of weaning from mechanical ventilation according to new categories
}

\author{
G-C. Funk*, S. Anders*, M-K. Breyer*, O.C. Burghuber*, G. Edelmann\#, W. Heindl*, \\ G. Hinterholzer ${ }^{\star}$, R. Kohansal*, R. Schuster ${ }^{+}$, A. Schwarzmaier-D’Assie $^{\S}$, \\ A. Valentin ${ }^{\S}$ and S. Hart|*
}

ABSTRACT: Weaning from mechanical ventilation was categorised as simple, difficult or prolonged by an international task force of the American Thoracic Society/European Respiratory Society/European Society of Intensive Care Medicine/Society of Critical Care Medicine/Sociéte de Réanimation de Langue Française in 2007. This new classification has not been tested in clinical practice. The objective of the present study was to determine the incidence and outcome of weaning according to the new categories.

We included medical and surgical patients who required mechanical ventilation in a prospective, multicentre, 6-month cohort study.

From an initial cohort of $\mathbf{5 1 0}$ patients, 257 intubated patients started weaning. Of these patients, the cumulative incidences of simple, difficult, and prolonged weaning were $152(59 \%), 68(26 \%)$ and $37(14 \%)$, respectively. Hospital mortality was increased in patients with prolonged (32\%) but not difficult $(9 \%)$ weaning in comparison with those with simple weaning $(13 \%)$, overall $p=0.0205$. In a multivariate logistic regression model, prolonged but not difficult weaning was associated with an increased risk of death. Ventilator-free days and intensive care unit (ICU)-free days were decreased in both difficult and prolonged weaning.

In conclusion, the new weaning category prolonged weaning is associated with increased mortality and morbidity in the ICU. The new category difficult to wean was associated with increased morbidity, but not mortality.

KEYWORDS: Epidemiology, extubation, intensive care unit

$\mathbf{T}$ he weaning process is a key element of mechanical ventilation, occupying up to $50 \%$ of its total duration [1]. It is crucial to identify the right time to extubate a patient, since re-intubation after pre-term extubation is associated with an increased risk for nosocomial pneumonia, prolonged intensive care unit (ICU) stay and death, and also accounts for substantially increased costs [2-4]. Conversely, an unnecessary delay in extubating a patient who has successfully passed a weaning test may also increase morbidity and mortality due to complications associated with prolonged mechanical ventilation [5-7].

In 2007, the European Respiratory Society (ERS), American Thoracic Society (ATS), European Society of Intensive Care Medicine (ESICM), Society of Critical Care Medicine (SCCM) and Societé de Réanimation de Langue Francaise (SRLF) held an International Conference on weaning from mechanical ventilation [8]. Therein, the spontaneous breathing trial was identified as the major diagnostic test to determine whether patients can be successfully extubated. Based on the overall duration of weaning as well as the number of spontaneous breathing trials required to liberate a patient from the ventilator, weaning was categorised into three groups (simple, difficult and prolonged weaning; table 1).

This new classification has not yet been tested in clinical practice, and the incidences of the new weaning categories remain unknown. Moreover, whether the classification correlates with patient mortality has not been investigated. Patients that experience simple weaning are known to have a good prognosis, with an ICU and hospital mortality of $11 \%$ and $19 \%$, respectively [6]. In contrast, the ICU mortality of patients failing the initial spontaneous breathing trial increases to $25 \%[6,7]$. Whether patients with prolonged or difficult

\section{AFFILIATIONS}

*Dept of Respiratory and Critical Care Medicine and Ludwig Boltzmann Institute for COPD, OttoWagner Hospital,

${ }^{\#}$ Dept for Anesthesia and Intensive Care Medicine, Krankenanstalt Rudolfstiftung,

${ }^{\S}$ II. Medical Dept, Krankenanstalt Rudolfstiftung,

I. Medical Dept,

Sozialmedizinisches Zentrum Süd -

Kaiser-Franz-Josef-Spital, and

${ }^{+}$I. Medical Dept,

Sozialmedizinisches Zentrum Ost -

Donauspital, Vienna, Austria.

\section{CORRESPONDENCE}

S. Hartl

Dept of Respiratory and Critical Care Medicine

Otto Wagner Spital

Sanatoriumstrasse 2

A-1140

Vienna

Austria

E-mail: sylvia.hartl@wienkav.at

Received:

April 052009

Accepted after revision:

June 092009

First published online:

July 022009

European Respiratory Journal

Print ISSN 0903-1936

Online ISSN 1399-3003 
weaning demonstrate a worse outcome than patients with simple weaning remains unknown, since applying the criteria of the new categorisation may prevent premature extubation and complications related to reintubation.

Since the new classification was based solely on expert opinion, it was noted in the panel pronouncement that "the proposed definitions of the weaning process and the groups of patients who undergo weaning need to receive careful testing and scrutiny. Studies of the patients' outcomes in the difficult weaning and prolonged weaning groups are needed" [8]. Therefore, we aimed to determine the incidence and outcome of weaning according to the new categories in a prospective cohort study. The hypothesis of the study was that outcome differs between the weaning categories. In the study, the weaning category represented the variable of interest, and hospital mortality the main outcome. Possible benefits from the new weaning categories would be related to a better prediction of patient outcome.

\section{METHODS}

\section{Study design and setting}

A prospective cohort study was conducted in five medicalsurgical ICUs at four different municipal hospitals in Vienna. Each ICU collected patient data during a 6-month period. The first patient was admitted to one of the participating ICUs on May 5, 2007 and the last patient was admitted on May 13, 2008.

\section{Patients}

All patients consecutively admitted to the ICU and requiring mechanical ventilation were screened. We excluded patients who received noninvasive ventilation without subsequent intubation, patients who died or were transferred to another ICU before they were ready to wean, and patients who experienced unplanned extubation before or during the weaning process. Finally, we included all consecutive patients who were weaned according to the recommendations [8] without unplanned extubation during the weaning phase (fig. 1).

\section{Baseline patient data}

The prospectively collected data included sociodemographic data (e.g. age, sex and comorbid conditions), reasons for ICU admission according to a predefined list of medical and

\begin{tabular}{l}
$\begin{array}{l}\text { TALE } 1 \text { Classification of patients according to the } \\
\text { weaning process }\end{array}$ \\
$\begin{array}{l}\text { Group/category } \\
\text { Definition }\end{array}$ \\
\hline Simple weaning \\
$\begin{array}{l}\text { Patients who proceed from the initiation of weaning } \\
\text { to successful extubation on the first attempt } \\
\text { without difficulty } \\
\text { Patients who fail initial weaning and require up to } \\
\text { three spontaneous breathing trials or as long as } 7 \\
\text { days from the first spontaneous breathing trial to } \\
\text { achieve successful weaning } \\
\text { Patients who fail at least three weaning attempts or } \\
\text { require }>7 \text { days of weaning after the first } \\
\text { spontaneous breathing trial }\end{array}$ \\
\hline
\end{tabular}

surgical diagnoses [9], and illness severity (as measured by the Simplified Acute Physiology Score (SAPS) II [10]). Data collectors coded diagnoses based on the documentation available in the patient's medical record.

\section{Weaning procedures}

All five ICU teams received detailed instructions regarding the weaning recommendations and were trained to follow the steps provided in a flowchart of the weaning process. Questions about the weaning procedures were encouraged and were answered by the authors (S. Anders, S. Hartl or W. Heindl) at any time before and during the study. While the entire medical team was instructed about the weaning procedure, one local study coordinator was responsible for data entry.

Weaning was conducted according to the current statement of the ERS, ATS, ESICM, SCCM and SRLF [8]. Weaning was considered as early as possible in the course of mechanical ventilation and involved a two-step strategy. First, readiness for weaning was assessed daily according to the criteria in the panel pronouncement [8]; patients who fulfilled these criteria underwent a spontaneous breathing trial as a diagnostic test to determine the likelihood of successful extubation. The initial spontaneous breathing trial lasted $30 \mathrm{~min}$ and consisted of breathing with a $\mathrm{T}$-tube or continuous positive airway pressure (CPAP; with $5 \mathrm{cmH}_{2} \mathrm{O}$ positive end-expiratory pressure (PEEP)). Alternatively a weaning trial using $5-8 \mathrm{cmH}_{2} \mathrm{O}$ pressure support with $5 \mathrm{cmH}_{2} \mathrm{O}$ PEEP was performed. When a patient successfully passed the spontaneous breathing trial according to the criteria in the panel pronouncement [8], extubation was suggested. The final decision to extubate was made by the physician in charge. When a patient failed the initial spontaneous breathing trial according to the criteria in the panel pronouncement [8], mechanical ventilation was reinstituted and the clinician reviewed the possible reversible aetiologies for the failure. The spontaneous breathing trial was repeated on the next day, if the patient was again ready-towean. A detailed description of the weaning process is available elsewhere [8].

A patient was rated as successfully weaned when he or she was extubated and was breathing spontaneously without any invasive or noninvasive ventilatory support for $\geqslant 48 \mathrm{~h}$. Patients who had undergone tracheotomy before they were ready to be weaned were considered successfully weaned when they were breathing spontaneously either through the tracheal cannula or directly through the tracheostoma for $48 \mathrm{~h}$ without any support [8]. If a patient was re-intubated after successful primary weaning and subsequently recommenced secondary weaning, only the primary weaning episode was used to define the patient's weaning category.

The total weaning duration was determined for patients who were successfully weaned at least once. The total weaning duration was calculated as the days between the time when a patient was first ready to wean and the time when a patient was successfully weaned for the last time.

\section{Outcome measures}

The primary outcome measure was vital status upon hospital discharge (hospital mortality). Secondary outcome measures 


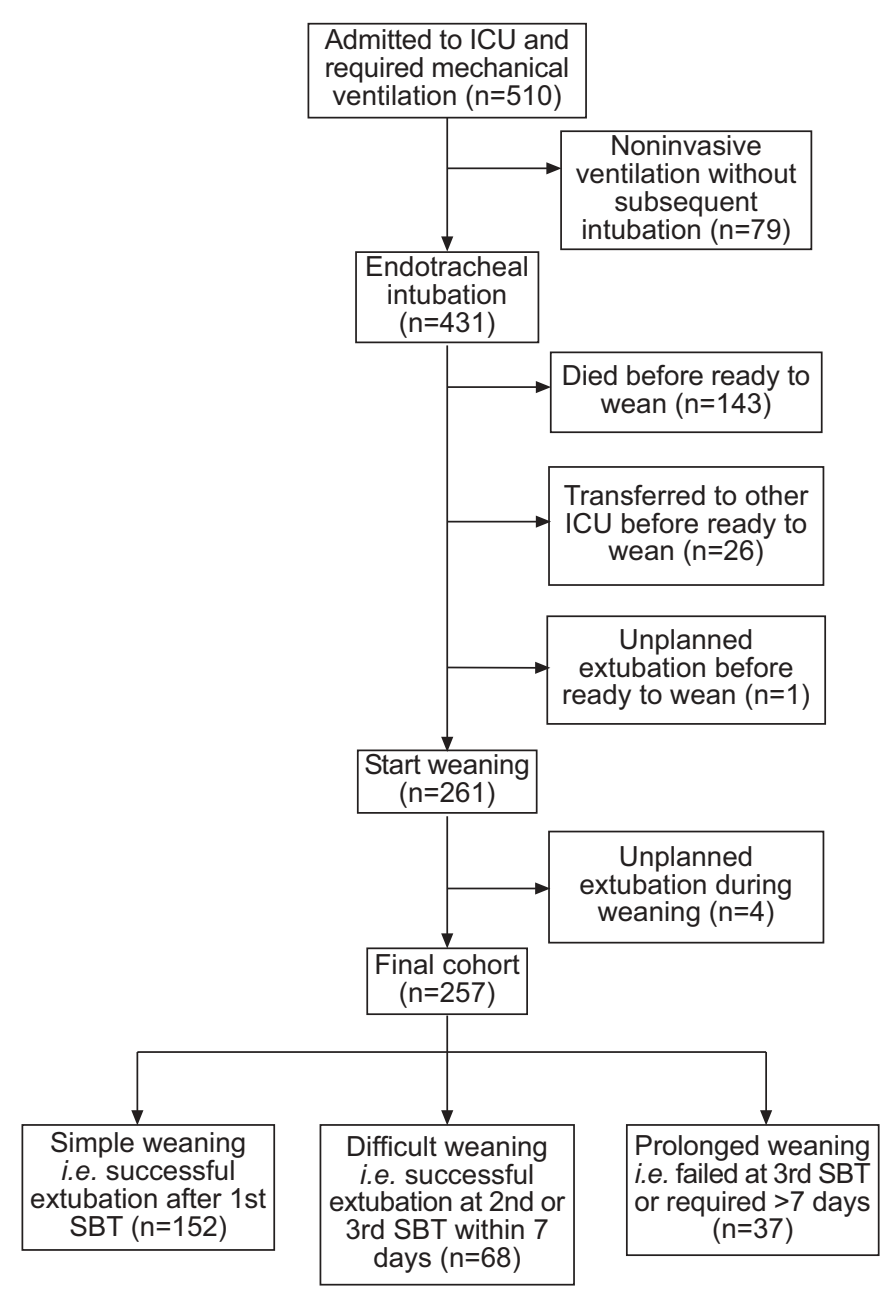

FIGURE 1. Study flow chart. ICU: intensive care unit; SBT: spontaneous breathing trial.

included the re-intubation rate, tracheotomy rate, number of ventilator-free days within 28 days after ICU admission, number of ICU-free days within 28 days after ICU admission, ICU mortality, post-ICU mortality, length of ICU stay and length of hospital stay.

\section{Data management}

Baseline patient data were documented on a printout case report form upon inclusion of a patient. Details about the weaning procedure were documented daily after spontaneous breathing trials. Outcome measures were documented by the local study coordinators together with a study assistant who visited each ICU every 2-3 weeks. All data were entered anonymously into a computer database (MS Office Access 2003 , Microsoft, Redmond, WA, USA) and assigned to individual record numbers. The study protocol was approved by institutional review. Since no additional interventions were performed and no individual data were analysed, the need for informed consent was waived.

\section{Statistical methods}

Statistical analyses were performed using SPSS version 13.0 software (SPSS Inc., Chicago, IL, USA). A Kruskal-Wallis
ANOVA was used for comparison of continuous variables with Dunn's test for post hoc testing. The Chi-squared test for trends was used to compare categorical variables between the three weaning categories. Multivariate logistic regression analysis was used to explore the association of the weaning category with hospital mortality. "Simple weaning" was defined as the reference category. Covariates were sequentially added to the logistic regression model if they were significant in the univariate analyses $(\mathrm{p}<0.2)$ and if they substantially altered the coefficient for either weaning category by an a priori defined level of $10 \%$ [11]. The sequences of covariates considered were based on the strength of the univariate association [11]. Finally, primary interaction terms for the weaning categories and the time between ICU admission and the start of weaning were included in the model. Data are presented as means $\pm \mathrm{SD}$, medians (1st to 3rd quartiles), or proportions, as appropriate. A two-sided p-value $<0.05$ was considered statistically significant.

\section{RESULTS}

We screened 510 patients who were admitted to the ICUs requiring mechanical ventilation. $79(15 \%)$ patients received noninvasive ventilation and did not require subsequent intubation. The remaining 431 (85\%) patients were intubated. Of the intubated patients, $170(39 \%)$ did not start weaning because of death (33\%), transfer to another ICU $(6 \%)$ or unplanned extubation $(0.2 \%)$. The remaining 261 (51\% of the initial cohort or $61 \%$ of the intubated patients) patients started weaning according to the recommendations. Four $(1 \%$ of all intubated patients) patients experienced unplanned extubation during weaning and were excluded from the analysis. Therefore, 257 patients were included in the final analysis. The study flow chart is shown in figure 1.

Among the patients included in the final analysis (i.e. those patients who underwent weaning according to the guidelines), the cumulative incidences of simple, difficult and prolonged weaning were 152 (59\%), 68 (26\%) and 37 (14\%), respectively. The baseline characteristics of the 257 patients stratified by weaning category are shown in table 2 . Age and SAPS II score at the time of admission did not differ between the groups. Comorbidity of chronic respiratory failure as well as a respiratory cause of admission was more common in patients with prolonged weaning as compared with those with simple weaning.

There was an overall difference in the proportion of patients successfully weaned between the weaning categories (simple weaning $98 \%$, difficult weaning $99 \%$, prolonged weaning $74 \%$; $\mathrm{p}<0.0001)$. However, the difference was only observed between simple or difficult weaning versus prolonged weaning (for both comparisons $\mathrm{p}<0.0001$ ), and not between simple versus difficult weaning $(\mathrm{p}=0.9998)$. The total weaning durations (median (interquartile range)) were $0.5(0.0-0.9), 2.9$ (2.63.5) and 10.0 (6.6-23.1) days in patients with simple, difficult and prolonged weaning, respectively (overall $\mathrm{p}<0.0001$, simple versus difficult $\mathrm{p}=0.037$; all other post hoc comparisons $\mathrm{p}<0.0001)$.

The overall ICU mortality and hospital mortality of patients who had undergone weaning according to recommendations were $5 \%$ and $14 \%$, respectively. The ICU mortality and hospital 
TABLE 2 Baseline characteristics of the 257 patients who started weaning, stratified by weaning category

\begin{tabular}{|c|c|c|c|c|}
\hline Subjects & $152(59)$ & $68(26)$ & $37(14)$ & \\
\hline Males & $81(53)$ & $33(49)$ & $22(59)$ & 0.8831 \\
\hline Number of comorbidities on ICU admission & $1(0-1)$ & $0(0-1)$ & $1(0-1.5)$ & 0.4341 \\
\hline \multicolumn{5}{|l|}{ Types of comorbidity } \\
\hline Chronic respiratory failure & $19(13)$ & $11(16)$ & $10(27)$ & $0.0346^{\circ}$ \\
\hline Chronic heart failure & $11(7)$ & $0(0)$ & $1(3)$ & 0.0616 \\
\hline Insulin-dependent diabetes & $8(5)$ & $3(4)$ & $5(14)$ & 0.1391 \\
\hline \multicolumn{5}{|l|}{ Types of ICU admission } \\
\hline Medical & $88(58)$ & $43(63)$ & $25(68)$ & 0.2348 \\
\hline \multicolumn{5}{|l|}{ Cause of admission } \\
\hline Gastrointestinal surgery & $10(7)$ & $10(15)$ & $5(14)$ & 0.0756 \\
\hline Cardiovascular surgery & $0(0)$ & $0(0)$ & $1(3)$ & 0.0479 \\
\hline Neurosurgery & $18(12)$ & $5(7)$ & $5(14)$ & 0.8977 \\
\hline Other types of surgery & $22(14)$ & $3(4)$ & $0(0)$ & $0.0019^{+}$ \\
\hline Cardiovascular disease & $21(14)$ & $8(12)$ & $4(11)$ & 0.5699 \\
\hline Neurological disease & $23(15)$ & $11(16)$ & $3(8)$ & 0.4038 \\
\hline Respiratory disease & $25(16)$ & $17(25)$ & $14(38)$ & $0.0038^{\S}$ \\
\hline
\end{tabular}

Data are presented as $n(\%)$, mean \pm SD or median (interquartile range), unless otherwise stated. ICU: intensive care unit; SAPS: simplified acute physiology score.

\#: simple versus difficult $p=0.1000$, simple versus prolonged $p=0.2053$, difficult versus prolonged $p=0.9999$; 9 : simple versus difficult $p=0.4859$, simple versus prolonged $p=0.0248$, difficult versus prolonged $p=0.1609$; $^{+}$: simple versus difficult $p=0.0298$, simple versus prolonged $p=0.0089$, difficult versus prolonged $p=0.5504$;

s: simple versus difficult $p=0.1358$, simple versus prolonged $p=0.0039$, difficult versus prolonged $p=0.1683$.

mortality of patients with simple, difficult and prolonged weaning are shown in table 3 . Both ICU and hospital mortality were higher for patients with prolonged weaning compared with patients with simple or difficult weaning; however, the ICU and hospital mortality of patients that experienced difficult weaning did not differ from those in patients with simple weaning (fig. 2). Post-ICU mortality did not differ between weaning categories (table 3). The days between ICU admission and the time at which patients started weaning significantly increased from simple to difficult to prolonged weaning (table 3).

Using simple weaning as the reference category, multivariate logistic regression identified prolonged weaning as an independent risk factor for hospital mortality. In contrast, difficult weaning was not associated with an increased risk for hospital mortality. Age was also identified as an independent risk factor of mortality. The study site was not significant in the regression. A trend toward a significant interaction was found between the weaning categories and the days between ICU admission and the time patients started weaning. The final model is shown in table 4 .

Patients who experienced difficult weaning had an increased length of hospital stay compared with patients with simple weaning. However, length of hospital stay did not further increase in patients with prolonged weaning (table 3). Both difficult and prolonged weaning significantly increased ICU length of stay and decreased the number of ICU-free and ventilator-free days within 28 days (table 3 and fig. 3). However, patients that experienced difficult weaning demonstrated fewer ICU-free days and ventilator-free days than patients with simple weaning. This was due primarily to a longer delay between ICU admission and "ready to wean" status, and was only secondarily due to a longer total weaning duration.

The rate of tracheotomy was increased in patients with prolonged weaning, but it did not differ between simple and difficult weaning groups (table 3). The proportion of patients who were re-intubated did not differ between the weaning categories (table 3).

The proportion of ICU survivors transferred to intermediate care units or normal wards did not differ between the weaning categories (table 3). Of the 220 patients who were discharged from the hospital, one patient remained on mechanical ventilation. This patient had experienced prolonged weaning.

\section{DISCUSSION}

We determined the incidence and outcome of weaning according to the new categories in a prospective cohort study. The main finding was that difficult weaning, although it 
TABLE 3 Incidence and outcome for the 257 patients who started weaning according to the new categories

\begin{tabular}{|c|c|c|c|c|}
\hline & Simple weaning & Difficult weaning & Prolonged weaning & p-value \\
\hline Subjects & $152(59)$ & $68(26)$ & 37 (14) & \\
\hline Post-ICU mortality & $14(10)$ & $5(7)$ & $4(14)$ & 0.7550 \\
\hline Hospital mortality & $19(13)$ & $6(9)$ & $12(32)$ & $0.0205^{\circ}$ \\
\hline Days between ICU admission and "ready to wean" & $0.5(0.0-4.6)$ & $4.3(1.8-9.8)$ & $11.0(4.8-15.6)$ & $<0.0001^{+}$ \\
\hline Number of ventilator-free days within 28 days & $26(18-27)$ & $21(12-24)$ & $1(0-11)$ & $<0.0001^{f}$ \\
\hline Number of ICU-free days within 28 days & $23(13-26)$ & $17(8-21)$ & $0(0-7)$ & $<0.0001^{\# \#}$ \\
\hline ICU length of stay & $4(1-9)$ & $11(7-20)$ & $27(18-37)$ & $<0.0001^{\# \#}$ \\
\hline Hospital length of stay & $22(10-39)$ & $33(22-59)$ & $44(32-81)$ & $<0.0001^{\oplus}$ \\
\hline ICU survivors transferred to an intermediate care unit & $25(17)$ & $12(18)$ & 8 (28) & 0.2526 \\
\hline
\end{tabular}

Data are presented as $\mathrm{n}(\%)$ and median (interquartile range), unless otherwise stated. ICU: intensive care unit. ${ }^{*}$ : simple versus difficult $\mathrm{p}=0.6688$, simple versus prolonged $p=0.0007$, difficult versus prolonged $p=0.0009$; ${ }^{\prime}$ : simple versus difficult $p=0.4272$, simple versus prolonged $p=0.0033$, difficult versus prolonged $p=0.0022$; ${ }^{+}$: simple versus difficult $p=0.0007$, simple versus prolonged $p<0.0001$, difficult versus prolonged $p=0.0068 ;{ }^{\text {s }}$ : simple versus difficult $p=0.0527$, simple versus prolonged $p<0.0001$, difficult versus prolonged $p<0.0001 ;{ }^{f}$ : simple versus difficult $p=0.006$, all other comparisons $p<0.0001 ;{ }^{\# \#}: p<0.0001$ for all comparisons; ${ }^{\text {"थ }}$ : simple versus difficult $p=0.007$, simple versus prolonged $p<0.0001$, difficult versus prolonged $p=0.569$.

decreases the number of ventilator- and ICU-free days compared with simple weaning, does not cause increased mortality. However, mortality increased in patients with prolonged weaning in comparison to both simple and difficult weaning. Prolonged but not difficult weaning was identified as an independent risk factor for increased hospital mortality.

This is the first study to apply the new recommendations for weaning from mechanical ventilation in a prospective patient cohort. A disease classification ideally predicts outcome, including mortality. However, to date, only a few studies about weaning have provided information regarding the relationship

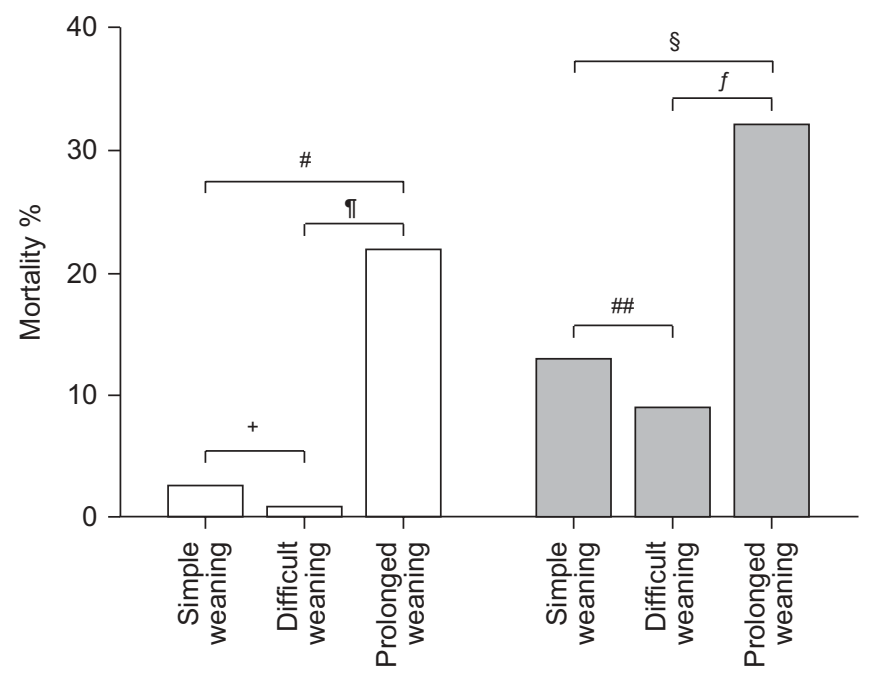

FIGURE 2. Intensive care unit $(\square)$ and hospital mortality ( $\square)$ stratified according to the weaning category. $n=257$. $^{*}: p=0.0007 ; " p=0.0009 ;^{+}: p=0.66 ;{ }^{\text {s }}: p=0.0033$; f: $p=0.0022 ; \# \#$ : $p=0.43$ between the difficulty of weaning and mortality. In a study performed by EsTEBAN et al. [6], patients who were successfully extubated after the first spontaneous breathing trial (i.e. simple weaning, according to the new classification) demonstrated an ICU mortality of $11 \%$. This result is comparable to our findings, taking into account that our patients were younger and had a higher prevalence of surgical admissions.

In two prior studies, the ICU mortality of patients who failed the first spontaneous breathing trial was $25 \%[6,7]$. These

\begin{tabular}{|c|c|c|c|}
\hline \multirow[t]{2}{*}{ TABLE 4} & \multicolumn{3}{|c|}{$\begin{array}{l}\text { Logistic regression model for the association of } \\
\text { the weaning categories with hospital mortality }\end{array}$} \\
\hline & & OR $(95 \% \mathrm{Cl})$ & $p$-value \\
\hline \multicolumn{4}{|c|}{ Weaning category } \\
\hline Simple we & & 1 (reference) & \\
\hline Difficult we & ning & $1.52(0.40-5.80)$ & 0.5448 \\
\hline Prolonged & reaning & $4.89(1.32-18.08)$ & 0.0171 \\
\hline $\begin{array}{l}\text { Days betwe } \\
\text { and the st }\end{array}$ & $\begin{array}{l}\text { ICU admission } \\
\text { rt of weaning days }\end{array}$ & $1.10(1.02-1.18)$ & 0.0123 \\
\hline \multicolumn{4}{|c|}{$\begin{array}{l}\text { ICU admission and the start of } \\
\text { weaning" by: }\end{array}$} \\
\hline Simple we & & 1 (reference) & \\
\hline Difficult we & ning & $0.86(0.72-1.02)$ & 0.0874 \\
\hline Prolonged & reaning & $0.91(0.82-1.01)$ & 0.0887 \\
\hline Age yrs & & $1.07(1.03-1.10)$ & $<0.0001$ \\
\hline SAPS II sco & points & $0.99(0.97-1.02)$ & 0.4793 \\
\hline
\end{tabular}

Data are presented as median (interquartile range). ICU: intensive care unit; SAPS: simplified acute physiology score. Hosmer and Lemeshow test Chisquared 10.18; $p=0.25$. 


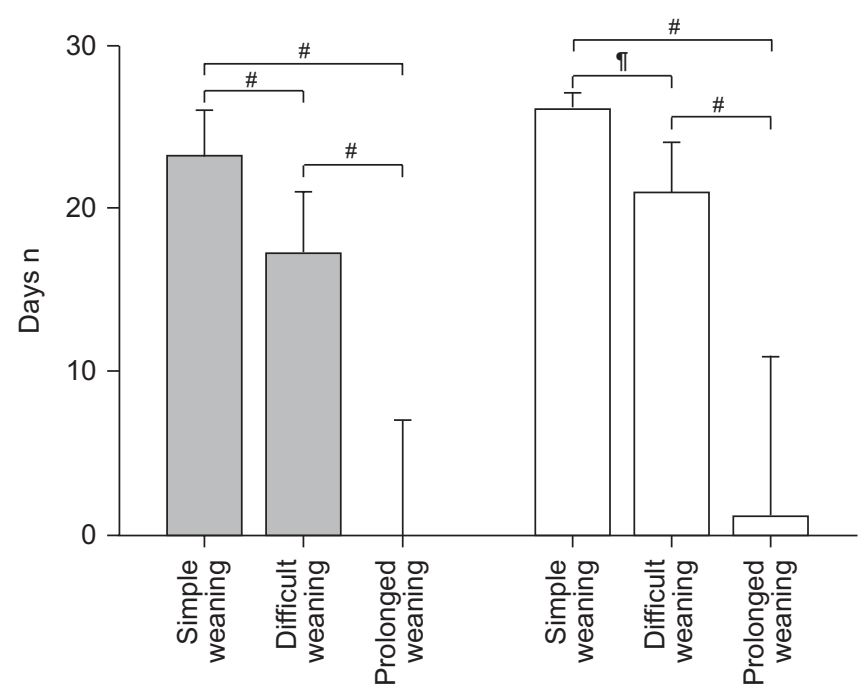

FIGURE 3. Number of intensive care unit- $(\square)$ and ventilator-free days $(\square)$ within 28 days stratified according to the weaning category. $n=257$. Bars indicate medians and whiskers indicate third quartiles. ${ }^{\#:} p<0.0001 ;{ }^{\circ}: p=0.006$.

patients comprised both difficult and prolonged weaning groups, according to the new classification. Hence, the results from these two prior studies cannot be directly compared with our data.

The finding that difficult weaning was not associated with mortality may be explained by both patient factors (e.g. differences in breathing patterns, underlying lung disease and sedation) and the accuracy of the ready to wean estimation in the clinical assessment.

The new weaning classification also correlated well with the number of ventilator- and ICU-free days. However, patients that experienced difficult weaning demonstrated fewer ICUand ventilator-free days than patients with simple weaning; this was mainly due to a longer delay between ICU admission and ready to wean status. Naturally, the definition of the weaning categories itself, which is based on an aspect of time (number of spontaneous breathing trials on different days) also contributed to this difference. Therefore, the category difficult weaning has only limited importance for the number of ventilator- and ICU-free days.

The reasons explaining why a patient experienced simple, difficult or prolonged weaning were not the primary interest of the study. The fact that the SAPS II score on admission was not associated with death may be explained by the significant proportion of patients that died before any weaning attempt was made. Thus, the power of the SAPS II score to predict death as associated with the weaning categories may have been weakened by the loss of the most severely ill patients.

The current findings may be important for conclusions drawn by intensive care physicians during the course of the weaning process. Failing the first three spontaneous breathing trials is associated with increased days spent in the ICU and an increased risk for mortality. If those patients with prolonged weaning survive, they will require a long period of time before they can be discharged from the hospital. This group of patients in particular may require and benefit from early and intensified physiotherapy and rehabilitation in the ICU. In the present study, we were unable to show that patients with prolonged weaning required transfer to intermediate care units more often. This might be due to the absence of predefined transfer criteria or the varying availability of resources in step-down units.

Patients with prolonged weaning more often underwent tracheotomy than did patients with simple or difficult weaning. Of note, a substantial proportion of patients underwent tracheotomy before weaning was initiated. The current guidelines define weaning success as extubation and the absence of ventilatory support $48 \mathrm{~h}$ following the extubation [8]. According to this definition, patients who breathe spontaneously through a tracheostomy tube without ventilatory support are not weaned, even though they may have required only one spontaneous breathing trial. In contrast, such patients were treated as being successfully weaned in the present study. The increasing use of early tracheotomy should be considered in future weaning classifications $[12,13]$. Early tracheotomy may also explain why 10 patients eventually classified as simple weaning underwent tracheotomy. Six of these patients had a neurological or neurosurgical cause of admission, which possibly caused the physician to expect prolonged weaning and initiate tracheotomy before the patients were ready to wean.

There are several limitations to the current study. We did not consider the use of noninvasive ventilation prior to intubation. Since all patients included finally required intubation, however, an effect of noninvasive ventilation prior to intubation on the course and outcome of weaning is unlikely. Secondly, only one of our patients was treated in the ICU after cardiovascular surgery. Therefore, no statement about this common type of ICU patient can be made. However, most patients that undergo cardiac surgery can be weaned from mechanical ventilation within a few hours after surgery [14], whereas only $\sim 5 \%$ require ventilator support for a duration of $\geqslant 7$ days [15-17]. Thirdly, information about the medical history of patients prior to the ICU was not systematically collected, but data entry was based on the information available upon ICU admission. Therefore, the prevalence of certain comorbidities may have been underestimated. This might explain the low prevalence of chronic heart failure and its absence as a risk factor associated with the weaning classification. Of note, it was not our intention to identify predictors of weaning success. Therefore, no information regarding the accuracy of weaning predictors (e.g. rapid shallow breathing index) is reported in this study.

As a further limitation, we cannot provide any information about the causes of death within the weaning categories. Since secondary weaning (i.e. weaning after re-intubation) was not considered in this study, the true incidence of prolonged weaning and its association with mortality may even be underestimated. Moreover, we cannot provide any exact information with regard to the costs of treatment in the weaning categories. Since prolonged weaning was associated with an increased number of ICU and ventilator days; however, it is likely that it also increases the cost of treatment. Finally, we only included a limited patient sample from a single city; therefore, our estimation of the epidemiology of prolonged weaning probably cannot be generalised. 
In conclusion, the new weaning category prolonged weaning is associated with increased mortality and morbidity in the ICU. The new category difficult to wean was associated with increased morbidity, but not mortality.

\section{SUPPORT STATEMENT}

The present study was supported by the Ludwig Boltzmann Institute for COPD (Vienna, Austria).

\section{STATEMENT OF INTEREST}

None declared.

\section{ACKNOWLEDGEMENTS}

The authors thank N. Dornhofer (Research Study Nurse at Ludwig Boltzmann Institute for COPD, Otto Wagner Hospital, Vienna, Austria) for excellent assistance with collecting, entering and editing of the data.

\section{REFERENCES}

1 Esteban A, Alia I, Ibanez J, et al. Modes of mechanical ventilation and weaning. A national survey of Spanish hospitals. The Spanish Lung Failure Collaborative Group. Chest 1994; 106: 1188-1193.

2 Torres A, Gatell JM, Aznar E, et al. Re-intubation increases the risk of nosocomial pneumonia in patients needing mechanical ventilation. Am J Respir Crit Care Med 1995; 152: 137-141.

3 Epstein SK, Ciubotaru RL, Wong JB. Effect of failed extubation on the outcome of mechanical ventilation. Chest 1997; 112: 186-192.

4 Seymour CW, Martinez A, Christie JD, et al. The outcome of extubation failure in a community hospital intensive care unit: a cohort study. Crit Care 2004; 8: R322-R327.

5 Coplin WM, Pierson DJ, Cooley KD, et al. Implications of extubation delay in brain-injured patients meeting standard weaning criteria. Am J Respir Crit Care Med 2000; 161: 1530-1536.

6 Esteban A, Alia I, Tobin MJ, et al. Effect of spontaneous breathing trial duration on outcome of attempts to discontinue mechanical ventilation. Spanish Lung Failure Collaborative Group. Am J Respir Crit Care Med 1999; 159: 512-518.
7 Vallverdu I, Calaf N, Subirana M, et al. Clinical characteristics, respiratory functional parameters, and outcome of a two-hour Tpiece trial in patients weaning from mechanical ventilation. Am J Respir Crit Care Med 1998; 158: 1855-1862.

8 Boles JM, Bion J, Connors A, et al. Weaning from mechanical ventilation. Eur Respir J 2007; 29: 1033-1056.

9 Metnitz PG, Steltzer H, Popow C, et al. [Definition and evaluation of a documentation standard for intensive care medicine: the ASDI (Working Group for Standardization of a documentation system for Intensive care medicine) pilot project]. Wien Klin Wochenschr 1997; 109: 132-138.

10 Le G Jr, Lemeshow S, Saulnier F. A new Simplified Acute Physiology Score (SAPS II) based on a European/North American multicenter study. JAMA 1993; 270: 2957-2963.

11 O'Brien JM Jr, Phillips GS, Ali NA, et al. Body mass index is independently associated with hospital mortality in mechanically ventilated adults with acute lung injury. Crit Care Med 2006; 34: 738-744.

12 Arabi Y, Haddad S, Shirawi N, et al. Early tracheostomy in intensive care trauma patients improves resource utilization: a cohort study and literature review. Crit Care 2004; 8: R347-R352.

13 Rumbak MJ, Newton $\mathrm{M}$, Truncale $\mathrm{T}$, et al. A prospective, randomized, study comparing early percutaneous dilational tracheotomy to prolonged translaryngeal intubation (delayed tracheotomy) in critically ill medical patients. Crit Care Med 2004; 32: 1689-1694.

14 Liu LL, Gropper MA. Respiratory and hemodynamic management after cardiac surgery. Curr Treat Options Cardiovasc Med 2002; 4: 161-169.

15 Pappalardo F, Franco A, Landoni G, et al. Long-term outcome and quality of life of patients requiring prolonged mechanical ventilation after cardiac surgery. Eur J Cardiothorac Surg 2004; 25: 548-552.

16 Yende S, Wunderink R. Causes of prolonged mechanical ventilation after coronary artery bypass surgery. Chest 2002; 122: 245-252.

17 Kern $\mathrm{H}$, Redlich $\mathrm{U}$, Hotz $\mathrm{H}$, et al. Risk factors for prolonged ventilation after cardiac surgery using APACHE II, SAPS II, and TISS: comparison of three different models. Intensive Care Med 2001; 27: 407-415. 